\title{
World osteoporosis day: celebrating two decades of progress in preventing osteoporotic fractures in Iran
}

\author{
Ali Tootee ${ }^{1} \cdot$ Bagher Larijani $^{2}$ (D) \\ Received: 28 October 2019 / Accepted: 4 November 2019/Published online: 26 November 2019 \\ (C) Springer Nature Switzerland AG 2019
}

Every year, World Osteoporosis Day is celebrated all across the world with the main objective of raising awareness and education of the general public, healthcare professionals, and policy-makers about a silent disease with devastating consequences which can be effectively prevented through lifestyle modification and early detection. This year's campaign is planned to be launched with the theme: "THAT'S OSTEOPOROSIS" which highlights the emotional burden imposed on people living with osteoporosis all across the world. Osteoporosis, which leads to decreased bone mass and increased risk of fractures, is the most common metabolic bone disease. Clinical consequences of the fractures which usually involve the spine, forearm and the hip can be grave, particularly in case of hip fractures which leads to grave health and financial consequences.

The burden of osteoporotic fracture has significantly increased all across the world. In the recent years, in most countries of the Middle East, levels of physical activity has significantly decreased, partially due to improvements in socioeconomic status of the citizens. This, consequently, has led to increased rates of osteoporosis and related fractures. Considering the fact that the proportion of the elderly population is forecast to significantly increase in this region of the world, the prevalence of osteoporotic fractures is expected to dramatically rise in the Middle East [1].

During the recent decades, global prevalence of osteoporosis has dramatically increased, primarily because of the aging populations. It is forecast that demographic changes in the

\section{Bagher Larijani}

emrc@tums.ac.ir

1 Diabetes Research Center, Endocrinology and Metabolism Clinical Sciences Institute, Tehran University of Medical Sciences, Tehran, Iran

2 Endocrinology and Metabolism Research Center, Endocrinology and Metabolism Clinical Sciences Institute, Tehran University of Medical Sciences, Tehran, Iran next 50 years will lead to an unprecedented increase in the prevalence of osteoporosis all across the globe. According to the results of research we've carried out at the Endocrinology and Metabolism Research Institute of Tehran University of Medical Sciences, in Iran, in the population over 50, 22.2\% of women and $11.0 \%$ of men suffer from osteoporosis [2]. In another study designed and carried out at our research institute in 2001, total years of life lost due to osteoporotic fractures was reported 32,375 years for hip fractures, 3493 years for vertebral fracture, and 158 years for forearm fractures. Moreover, we observed that and osteoporosis was responsible for 36,027 year of life lost as a result of premature mortality and disability [3].

Vitamin D deficiency has been extensively spotlighted in the literature as one of the main causes of reduced bone mineral density and osteoporosis [4]. In Iran, the results of an extensive research we designed and carried out in 2004 in a nationally-representative sample population of 1210 subjects aged 20 to 64 demonstrated that $81.3 \%$ of the population had some degrees of vitamin D insufficiency, and the prevalence of the severe and moderate forms of this osteoporosis contributing factor were $9.5 \%$ and $57.6 \%$ respectively. It is noteworthy that in our study, neither sun exposure nor clothing habits were identified as predictors of vitamin D deficiency [5]. These results motivated us to design and implement several fortification projects in collaboration with the food industry.

As this silent, common, and potentially devastating disease often remains undiagnosed, it is of crucial importance do develop clinically-applicable tools for calculation of the osteoporotic fractures risk in healthy individuals [6]. Accordingly, FRAX algorithm has been developed to assess the probability of osteoporotic fracture in an individual within a given time period [7]. This algorithm which is specifically designed for primary care settings, calculates probability of osteoporotic fractures through measurement of several clinical risk factors both in men and women [8]. It should be noted that as different nations in various regions of the world significantly differ in terms of race-related anthropometric parameters, FRAX needs 
to be specifically modified and calibrated to be reliably employed in different countries. Considering this, at the EMRI, we have developed an Iranian-calibrated version of the FRAX tool which can be reliably utilized for calculation of fracture risk in Iranian population [9]. We have even proposed University of Sheffield additional parameters which may be integrated into the standard FRAX for potential enhancement of its cross-national practical utility.

Hip fractures, which are the most serious complications of osteoporosis, usually occur following a fall. The incidence of hip fractures dramatically increases as the age advances, and the risk of death is greatest in the first 6 months after the fracture. As bone loss and falls are more common in women than men, the incidence of hip fracture in twice more common in females [10]. Similar to other complex chronic diseases, the pathogenesis of hip fractures is multifactorial, and there exists no single factor to be blamed as the cause [10]. In Iran, according to the results of a nationwide research carried out at the Endocrinology and Metabolism Research Institute, approximately 8000 hip fractures occur every year [11]. Correspondingly, with the main objective of minimizing hip fractures and improvement of outcomes after fractures, we have developed a National Hip Fracture Register [12]. Besides clinical applications, data collected by the Iranian National Hip fracture Registry will be of great importance for future research in the field.

Vertebral fractures are also common, and they can lead to back pain, kyphosis, and height loss. It should be noted, however, that many vertebral fractures occur without pain. Therefore, as a considerable proportion of vertebral fractures do not have any clinical manifestation, and as there is no universally accepted definition for vertebral fracture, the epidemiology of vertebral fractures is not yet well elucidated. It is documented that only approximately a third of all vertebral fractures noted on radiographs are clinically detected. It is noteworthy that only a quarter of vertebral fractures are caused by falls, and most of them are ensue routine everyday activities such as bending or lifting light-weight objects. It should be also noted that a national register for spinal cord injury is being developed in Iran with the main objective of assessment of quality of care, long-term clinical outcomes and personal and psychological burden of traumatic spinal cord injury [13].

For accurate estimation of the national burden of osteoporosis, establishment of a nationwide osteoporosis network is of crucial importance. In Iran, Iranian Osteoporosis Research Network was developed by the Endocrinology and Metabolism Research Institute (EMRI) in 2002 [14]. Mounting public awareness campaigns, development of joint research projects, and establishment of a nationally-inclusive Hip Fracture Registry are amongst achievements this national network. Every year, several nationally-synchronized public awareness activities are designed and implemented by the Iranian National Osteoporosis Network on the occasion of the World Osteoporosis Day. Besides such public education activities, a number of professional education events (such as workshops, seminars, conferences...) are regularly organized and held by the Iranian Osteoporosis Network. It should be added, moreover, that EMRI, the coordinator of this network, is a WHO Collaborating Center on Research and Management of Osteoporosis, and has developed extensive regional and international networks as fulfillment of its commitments to the WHO.

With the main objective of delivering osteoporosis care services, several osteoporosis specialty clinics are developed by the EMRI. The first Osteoporosis Specialty clinic was established at the Shariati Hospital Campus (An educational hospital of the Tehran University of Medical Sciences) in 2001. Today several osteoporosis specialty clinics are operating all across Iran which exclusively provide osteoporosis care. In this national network of osteoporosis clinics, clinical services are exclusively provided for patients with osteoporosis and related bone diseases. Moreover, these clinics assist patients with low bone density to cope and reduce their risk of future fractures. As post-menopausal women are at greater risk of osteoporotic fractures, most patients referred to our osteoporosis clinics are women over 50. However, men and those with complex medical problems that put them at a greater risk for bone loss and fractures are regularly referred to us. The data collected in these clinics are also of great utility for design and implementation of national-scale multi-centric research projects by the Institute.

Osteoporosis is a growing global plight without any boundaries, and worldwide, osteoporotic fractures inflict one in three women and one in five men older than 50. This silent disease poses great health and financial burdens of health systems in both developing and developed countries. Therefore, development of national visionary and long-term prevention strategies is of crucial importance for combatting this immense threat and the enhancement of the health and wellbeing of the society. This goal can only be achieved through sustained and coordinated national and international efforts. With such political efforts and national commitment, osteoporosis would no longer be considered an inevitable consequence of aging.

\section{Compliance with ethical standards}

Conflict of interest BL is the head of Endocrinlogy and Metabolism Research Institute.

\section{References}

1. Maalouf G, et al. Middle East and North Africa consensus on osteoporosis. J Musculoskelet Nueronal Interact. 2007;7(2):131.

2. Khashayar P, et al. The prevalence of osteoporosis in an Iranian population. J Clin Densitom. 2010;1(13):112. 
3. Abolhassani F, Mohammadi M, Soltani A. Burden of osteoporosis in Iran. Iran J Public Health. 2004;33(Supple 1):18-28.

4. Holick MF. Vitamin D deficiency. N Engl J Med. 2007;357(3): 266-81.

5. Hashemipour S, et al. Vitamin D deficiency and causative factors in the population of Tehran. BMC Public Health. 2004;4(1):38.

6. Compston J. Assessment of fracture risk and its application to screening for postmenopausal osteoporosis (WHO Technical Report Series No 843). Ann Rheum Dis. 1995;54(7):548.

7. Kanis J, et al. FRAX ${ }^{\mathrm{TM}}$ and the assessment of fracture probability in men and women from the UK. Osteoporos Int. 2008;19(4):385-97.

8. Kanis JA, et al. Interpretation and use of FRAX in clinical practice. Osteoporos Int. 2011;22(9):2395.

9. Ghafoori S, et al. The risk of osteoporotic fractures and its associating risk factors according to the FRAX model in the Iranian patients: a follow-up cohort. Journal of Diabetes \& Metabolic Disorders. 2014;13(1):93.
10. Cummings SR, et al. Risk factors for hip fracture in white women. N Engl J Med. 1995;332(12):767-74.

11. Maalouf G, et al. Middle East and North Africa consensus on osteoporosis. J Musculoskelet Neuronal Interact. 2007;7(2):131-43.

12. Moayyeri A, et al. Epidemiology of hip fracture in Iran: results from the Iranian Multicenter Study on Accidental Injuries. Osteoporos Int. 2006;17(8):1252-7.

13. Sharif-Alhoseini, M., et al., National Spinal Cord Injury Registry of Iran (NSCIR-IR)-a critical appraisal of its strengths and weaknesses. Chinese Journal of Traumatology, 2019.

14. Meybodi HA, et al. Iranian osteoporosis research network: background, mission and its role in osteoporosis management. Iran $\mathrm{J}$ Public Health. 2008;37(supp):1-6.

Publisher's note Springer Nature remains neutral with regard to jurisdictional claims in published maps and institutional affiliations. 